\title{
The Local Lorentz Symmetry Violation and Einstein Equivalence Principle
}

\author{
Baocheng Zhang* \\ School of Mathematics and Physics, \\ China University of Geosciences, Wuhan 430074, China
}

\begin{abstract}
Lorentz symmetry violation (LV) was recently proposed to be testable with a new method, in which the effect of the violation is described as a certain local interaction [R. Shaniv, et al, PRL 120, 103202 (2018)]. We revisit this LV effect in the paper and show that it is not only local, but it also represents a classical violation according to the recent quantum formulation of the Einstein equivalence principle (EEP). Based on a harmonically trapped spin- $1 / 2$ atomic system, we apply the results of table-top experiments testing LV effect to estimate the corresponding violation parameter in the quantum formulation of EEP. We find that the violation parameter is indeed very small, as expected by the earlier theoretical estimation.
\end{abstract}

*Electronic address: zhangbc.zhang@yahoo.com 


\section{INTRODUCTION}

Invariance under Lorentz transformations constitutes one of the fundamental principles of modern physics. A more fundamental theory including a kind of quantum theory for gravity, however, implies the possibility or even necessity for Lorentz symmetry violation (LV) «13]. The study about this violation has attracted extensive attention over the past twenty years, and a nice theoretical framework called the Standard-Model Extension (SME) [4 6] was developed for the analysis of LV. This framework formally contains all possible Lorentzsymmetry breaking terms which are generated by the couplings between the standard fields and vacuum expectations of the tensor fields which parameterize symmetry violations (more definitely, it concerns only the violation of invariance under particle Lorentz transformations, see the Ref. [5]). The violation is always studied by using certain terms in the presumed absence of all other symmetry breaking terms.

Although the Lorentz symmetry remains a property of the underlying fundamental theory [5], it might be violated at experimentally accessible energy scales due to spontaneous symmetry breaking, hence the development of experimental tests for Lorentz invariance [715]. Of particular relevance to this aspect are several recent efforts using atoms and ions to test LV through precision measurements. For the LV term that we are interested in, it is usually tested by the isotropy of the speed of light (for recent results, please refer to Ref. [10]). In this direction, the most sensitive tests made use of neutral Dy atoms [11], $\mathrm{Ca}^{+}$ions [13], and $\mathrm{Yb}^{+}$ions [14]. A new method employing dynamic decoupling was suggested to be implementable in current atomic clock experiments [16], with which an especially high sensitivity to LV is predicted. In this recent proposal [16], if the total angular momentum of a physical system is fixed, the LV term can be regarded as an equivalent term proportional to the square of the $z$-component of total angular moment operator, a typical local interaction. In this paper, we will investigate the related effect from such an term. Apart from revisiting this issue in the current studies, we attempt to elucidate the meaning of locality clearly.

This interesting LV term concerns the dependence of physical results on the moving direction and the momentum of a particle, resulting in the violation of local Lorentz invariance (LLI) [17], which also represents the violation of Einstein equivalence principle (EEP) [18, 19]. Since the LV effect is always studied in those experiments made with the quantum systems even using the obvious quantum property (i.e. entanglement in Ref. [13, 14]), we 
hope to investigate whether the above mentioned LV effect is classical or quantum. This could be done by the recent suggestion about a quantum formulation of EEP [20, 21], in which the violations of LLI can happen as either classical or quantum effects but a criteria was given to distinguish them.

This paper is organized as follows. First, we revisit the LV term and reveal its relation to spatial anisotropy in the second section. This is followed by an analysis of it as a type of local interaction in the third section. In the fourth section, we compared this LV term with the description about LLI in the quantum formulation of EEP, and we also study the property of LV effect and estimate the corresponding violation parameter of LLI according to the related LV experiments. Finally, we end with a conclusion in the fifth section.

\section{LORENTZ SYMMETRY VIOLATION (LV)}

Start with the action of SME for QED [22],

$$
S_{S M E}=\int \mathcal{L}_{S M E} d^{4} x
$$

where $\mathcal{L}_{S M E}=\mathcal{L}_{\psi}+\mathcal{L}_{A}+\mathcal{L}_{g} . \mathcal{L}_{\psi}$ is the Lagrangian density of the fermion part and contains terms dominating at low energies that involve ordinary matter such as protons, neutrons and electrons and their minimal coupling with gravity and gauge particles, $\mathcal{L}_{A}$ contains gauge fields for the photon and the fields describing particles that are not 'ordinary', like muons, mesons, neutrinos and so on and the minimal couplings to gravity, and $\mathcal{L}_{g}$ is the gravitational part that includes the terms consisting of the vierbein and the spin connection. When LV is considered for the electron sector within the framework of SME, the Lagrangian density we are concerned is the fermion part (i.e. electron) up to the first-order expansion $[4,[5]$,

$$
\mathcal{L}_{\psi}=\frac{1}{2} i \bar{\psi}\left(\gamma_{\nu}+c_{\mu \nu} \gamma^{\mu}\right) \overleftrightarrow{D^{\nu}} \psi-\bar{\psi} m_{e} \psi
$$

where $m_{e}$ denotes the electron mass, $\psi$ is a Dirac spinor, $\gamma^{\mu}$ are the usual Dirac matrices

and $\bar{\psi} \overleftrightarrow{D^{\nu}} \psi \equiv \bar{\psi} D^{\nu} \psi-\psi D^{v} \bar{\psi}$ with $D^{v}$ the shorthand for the covariant derivative. When the other backgrounds of spacetime like Riemann-Cartan spacetime is considered, the vierbein and spin connection would enter into the Eq. (22), i.e. see Ref. [22] for the complete expression. The $c_{\mu \nu}$ tensor in the above Eq. (2) quantifies the strength of LV for the electron sector by the frame dependent interaction term, which gives an energy level shift 
[7, 24, 25],

$$
\delta H_{\mathrm{LSV}}=-\left[C_{0}^{(0)}-\frac{2 U}{3 c^{2}} c_{00}\right] \frac{\mathbf{p}^{2}}{2}-\frac{C_{0}^{(2)} T_{0}^{(2)}}{6} .
$$

where $\mathbf{p}$ is the momentum of the (lone) bound (valance) electron, $U$ is the Newtonian gravitational potential and the specific parameters $C_{0}^{(0)}$ and $c_{00}$ quantifying the strength of LV have been discussed and tested before [11]. It represents the violation of Lorentz symmetry and EEP in bound electronic states. The violation of EEP within SME frame stemmed from the dependence of effective mass on the gravitational potential and can be tested using the transition between atomic energy levels. In particular, the kinetic energy $\frac{\mathbf{p}^{2}}{2}$ would also be changed from one energy level to another one [15]. But in several other experiments, the different spin states are considered for the test of LV [13, 16] and the kinetic energy remains fixed when the spin states are changed. Thus, the first terms in Eq. (3) would not be observed in these experiments. From the perspective of EEP, the first term represents the violation of local position invariance (LPI) which usually tested by the experiments of gravitational redshift [23]. The second term represents the violation of local Lorentz symmetry.

The relativistic form of rank 2 irreducible tensor operator $T_{0}^{(2)}$ is $T_{0}^{(2)}=c \gamma_{0}\left(\gamma \mathbf{p}-3 \gamma_{z} p_{z}\right)$, with $p_{z}$ the momentum component along the quantization axis fixed in the laboratory frame. Its non-relativistic form becomes $T_{0}^{(2)}=\left(\mathbf{p}^{2}-3 p_{z}^{2}\right) / m_{e}$, where $p_{z}$ is the component of the electron's momentum along the quantization axis which is fixed in the laboratory, $m_{e}$ is the mass of the electron. Thus the second LV term $\propto C_{0}^{(2)}$ of $\delta H_{\mathrm{LSV}}$ reduces to,

$$
\delta H=-C_{0}^{(2)} \frac{\left(\mathbf{p}^{2}-3 p_{z}^{2}\right)}{6 m_{e}}
$$

where the parameter $C_{0}^{(2)}$ characterizes the violation of Lorentz symmetry we focus on. In relativistic physics, Lorentz symmetry implies an equivalence of observation or observational symmetry due to special relativity, or stated more formally that the laws of physics stay the same for all observers moving with constant velocities with respect to one another within an inertial frame. It has also been described sometimes as the independence of all experimental results on the orientation or the boost velocity of the laboratory through space [17], which is seen evidently in Eq. (4)

The matrix element of the $T_{0}^{(2)}$ operator is calculated using the Wigner-Eckart theorem 
as [13],

$$
\left\langle J, m\left|T_{0}^{(2)}\right| J, m\right\rangle=\frac{-J(J+1)+3 m^{2}}{\sqrt{(2 J+3)(J+1)(2 J+1) J(2 J-1)}}\left\langle J\left|T^{(2)}\right| J\right\rangle,
$$

where $J$ and $m$ denote the quantum numbers of the total electronic angular momentum and its projection along the quantization axis, respectively. The term proportional to $\mathrm{m}^{2}$ can be taken as a part of the signal for LV. If a physical system with a fixed total angular momentum $J$ is considered, as for the case first suggested in Ref. [16], the dynamics of LV term we discuss is described equivalently by the Hamiltonian

$$
H_{V}=\kappa J_{z}^{2}
$$

which is the very term we are concerned in this paper. In the following two sections, we will show that this kind of interaction is local and represents only a kind of classical violation based on the quantum formulation of EEP.

\section{LOCAL INTERACTION}

In earlier studies on the LV effect, different eigenstates with distinct absolute values of the angular momentum $J_{z}$ are chosen in order to extract the relative phase from a coherent superposition state of the eigenstates, i.e. in Ref. [16], the selected states are $\left|\frac{7}{2},-\frac{7}{2}\right\rangle$ and $\left|\frac{7}{2},-\frac{1}{2}\right\rangle$. The phase measurement is implemented through the influence of the LV term on Ramsey interferometry augmented by dynamical decoupling (DD).

The standard Ramsey interferometry consists of three parts: two $\frac{\pi}{2}$ pulses, and a free evolution in between. In terms of the pseudo-spin angular momentum operators, its time evolution is described as

$$
U_{\phi}=e^{-i \pi J_{x} / 2} e^{i \phi J_{z}} e^{i \pi J_{x} / 2}=e^{-i \phi J_{y}}
$$

where the two $\frac{\pi}{2}$ pulses act as 50:50 beamsplitters, and the middle term $e^{i \phi J_{z}}$ denotes the free evolution or the free rotation circling the z-axis (defined by a polarizing magnetic field) over an angle $\phi$. When the LV term is present, it adds to the above free evolution and gives

$$
U_{V \phi}=e^{-i \pi J_{x} / 2} e^{i \phi J_{z}} e^{-i \kappa t J_{z}^{2}} e^{i \pi J_{x} / 2}=e^{-i \phi J_{y}-i \kappa t J_{y}^{2}},
$$

which is analogous to the nonlinear Ramsey interferometer [26-28] and provided a way for estimating the LV parameter $\kappa$. 
The effect discussed above is generally interpreted as a local (to the atom considered) interaction. For an ensemble of $N$ atoms, the LV Hamiltonian can be expressed as

$$
H_{V}=\kappa J_{1 z}^{2}+\kappa J_{2 z}^{2}+\cdots+\kappa J_{N z}^{2}
$$

which leads to

$$
U_{V \phi}=\sum_{i=1}^{N} e^{-i \phi J_{y i}-i \kappa t J_{y i}^{2}} .
$$

Evidently, this remains local, as it cannot generate any coherent results among atoms. Consequently, such a form of local unitary interaction cannot alter entanglement of the quantum state for the ensemble [29]. This also means that although the operation described by $H_{V}$ is for an ensemble of $N$ atomic spins, the uncertainty in evaluating $\kappa$ scales as $\propto 1 / \sqrt{N}$, or follows the standard quantum limit (SQL). It would be desirable to develop ideas based on entangled quantum states, such as spin squeezed state [30, 31], the twin-fock state atomic Bose-Einstein condensate (BEC) [32 36], the ground state of an antiferromagnetic spin-1 atomic condensate [37, 38], and so on for improved scaling beyond the SQL.

Since the LV effect described by $H_{V}$ is local, it implies that this effect might represent only a type of classical violation although the discussion above is made under a quantum evolution. In the next section, we will investigate it using a recent framework developed for testing the Einstein equivalence principle (EEP) [20].

\section{CLASSICAL VIOLATION}

With the development of atomic precision measurements, some classical physical effects that are minute and difficult to be observed, such as the violation of weak equivalence principle (WEP), has been measured experimentally using quantum systems. Naturally, one wants to understand whether the influence or violation of these classical effects like WEP is actually caused by the quantum property. To address this, Zych and Brukner [20] constructed a new framework to test the quantum aspects of the EEP. For a non-relativistic

quantum system with its Hamiltonian given by $H_{\mathrm{nr}}=m c^{2}+\frac{P^{2}}{2 m}+m U(Q)$, where $m$ is the mass of the system, $Q, P$ are position and momentum operators, respectively, for the center of mass, and $U$ denotes the gravitational potential. In order to distinguish the influences of different internal energies on the EEP, they suggested a quantum formulation of the 
mass-energy equivalence principle by extending the mass expression $m$ in $H_{\mathrm{nr}}$ into

$$
M_{k}=m_{k} I^{\mathrm{int}}+\frac{H_{k}^{\mathrm{int}}}{c^{2}}
$$

where $k=\{r, i, g\}$ represents quantities related to rest, inertial, and gravitational masses, respectively, $I^{\text {int }}$ is the identity operator in the space of internal degrees of freedom, $H^{\text {int }}$ is the internal energy operator that can contribute to the mass, and $m$ is the mass of the corresponding ground state for $H^{\text {int }}$. Thus, the total Hamiltonian up to the lowest order in relativistic corrections is expressed as

$$
H_{\text {test }}=m_{r} c^{2}+H_{r}^{\mathrm{int}}+\frac{P^{2}}{2 m_{i}}+m_{g} U(Q)-H_{i}^{\mathrm{int}} \frac{P^{2}}{2 m_{i}^{2} c^{2}}+H_{g}^{\mathrm{int}} \frac{U(Q)}{c^{2}},
$$

where the validity of the EEP is guaranteed by $H_{r}^{\text {int }}=H_{i}^{\text {int }}=H_{g}^{\text {int }}$ or $M_{r}=M_{i}=M_{g}$. Actually, according to the purpose of measurements, the EEP can be divided into three classes. One is the validity of WEP which requires the condition $M_{i}=M_{g}$, or $H_{i}^{\text {int }}=H_{g}^{\text {int }}$ if the classical WEP holds by $m_{i}=m_{g} . H_{r}^{\text {int }}=H_{i}^{\text {int }}$ indicates the validity of LLI and $H_{r}^{\text {int }}=$ $H_{g}^{\text {int }}$ indicates the validity of LPI. Therefore, if the classical WEP is not violated, the violation from any one condition does not imply the violation of any other one, but once any two of the three conditions is broken, the third must be broken. Comparing Eq. (3) and Eq. (12), it is seen easily that the reasons of the violation of LPI are different. The former violation in Eq. (3) stemmed from the anomalous coupling between the gravitational potential and the external kinetic energy, and the latter violation in Eq. (12) is due to the anomalous coupling between the gravitational potential and the internal energy levels. So in the future, it deserves to make a further investigation for the possible Lorentz-breaking modifications in the presence of gravity from the perspective of experimental observation, since EEP is the concept of general relativity in essence. However, the related experiments [16] involved in this paper cannot be discussed for the violation of LPI under the background of quantum formulation of EEP [20]. Further, it is noted that the violation of LLI, irrespective of that described in Eq. (4) from SME or in Eq. (12) from quantum formulation of EEP, is both derived from the anomalous coupling between internal energy levels and the external kinetic energy. Thus, in order to study the behaviour of LV using the quantum formulation of EEP, it is convenient to assume that LPI is valid since the violation of LLI does not imply the violation of LPI. 
LLI can be tested with such a Hamiltonian [20],

$$
H_{L L I}=m_{r} c^{2}+H_{r}^{\mathrm{int}}+\frac{P^{2}}{2 m_{i}}-H_{i}^{\mathrm{int}} \frac{P^{2}}{2 m_{i}^{2} c^{2}},
$$

which constitutes an important part of the test framework for the EEP and the violation is caused only by $H_{r}^{\text {int }} \neq H_{i}^{\text {int }}$ or $M_{r} \neq M_{i}$. The terms related to the gravitational potential in Eq. (12) are ignored because they contribute equally to the evolution of different energy levels (that means these terms would be subtracted when calculating the difference of energy levels) if the LPI preserves.

After the test of the quantum aspects of EEP was suggested, some feasible experiments were also analyzed [21] or made [39]. In Ref. [21], a harmonically trapped spin- $\frac{1}{2}$ atom is proposed to test the Hamiltonian $H_{\text {test }}$ and the crucial point lies in the form

$$
H_{k}^{\mathrm{int}}=H_{r}^{\mathrm{int}}+\mu B \xi_{k}\left(H_{r}^{\mathrm{int}}\right), \quad \xi_{k}\left(H_{r}^{\mathrm{int}}\right)=\left(\begin{array}{cc}
a_{k} & b_{k} \\
b_{k}^{*} & c_{k}
\end{array}\right)
$$

where $k=i, g$ represents the violation of LLI, LPI, $B$ is the external magnetic field, and $\mu$ is the atomic magnetic moment. $H_{r}^{\text {int }}=\frac{1}{2} \mu B\left(\left|+\frac{1}{2}\right\rangle\left\langle+\frac{1}{2}|-|-\frac{1}{2}\right\rangle\left\langle-\frac{1}{2}\right|\right)=\mu B\left(\begin{array}{cc}\frac{1}{2} & 0 \\ 0 & -\frac{1}{2}\end{array}\right)$ and $\xi_{k}\left(H_{r}^{\text {int }}\right)$ quantifies the deviation from the EEP, and in particular, $\xi_{i}\left(H_{r}^{\text {int }}\right)$ quantifies the deviation from the LLI, but the required measurement was designed [21] in the presence of gravitation and external magnetic field. When the off-diagonal terms $b_{k}$ and its complex conjugate are not assumed to exist, the violation of EEP parameterized by $\xi_{k}$ is called the classical violation; otherwise, the violation is regarded as quantum.

It is noted that $H_{k}^{\text {int }}$ is actually not the test Hamiltonian, and the complete Hamiltonian we need is obtained by inserting $H_{i}^{\text {int }}$ into the Eq. (13),

$$
H_{L L I}=\frac{P^{2}}{2 m_{i}}+H_{r}^{\mathrm{int}}-H_{i}^{\mathrm{int}} \frac{P^{2}}{2 m_{i}^{2} c^{2}}=\frac{P^{2}}{2 m_{i}}+\mu B\left(\begin{array}{cc}
\frac{1}{2} & 0 \\
0 & -\frac{1}{2}
\end{array}\right)+\frac{P^{2}}{2 m_{i}^{2} c^{2}}\left(\begin{array}{cc}
a_{i}^{\prime} & b_{i}^{\prime} \\
b_{i}^{\prime *} & c_{i}^{\prime}
\end{array}\right)
$$

where the static mass term has been ignored since it contributes only a unrelated constant phase for the evolution of atoms. From the Eq. (15), the first and second terms indicate that the atom moves in a magnetic field freely and the $z$ direction of atomic spin is consistent with that of the magnetic field. For example, the atom with spin-up (the eigenvalue of the spin- $z$ component is $\frac{1}{2}$ ) state initially will keep in this state during the later evolution if no extra interaction is added. The third term represents the violation of LLI. Different 
from Ref. [21], we delete the " $\mu B$ " in the third term since no reason to imply that the assumption of LLI violation should be related to the presence of the external magnetic field. In other words, the term " $\mu B$ " is absorbed to the definition of the violation parameters $a_{i}^{\prime}$ $\left(=\left(a_{i}+\frac{1}{2}\right) \mu B\right)$ and $c_{i}^{\prime}\left(=\left(c_{i}-\frac{1}{2}\right) \mu B\right)$. For the off-diagonal terms in the third term of Eq. (15), it will lead to the change of the initial spin state, i.e. the atom with spin-up state initially will change into spin-down state (the eigenvalue of the spin- $z$ component is $-\frac{1}{2}$ ). For the diagonal term, it will lead to the shift of energy level. The corresponding shift of energy level induced by $\xi_{i}\left(H_{R}^{\mathrm{int}}\right)$ has been calculated and can be approximated as [21]

$$
\Delta E_{i}^{L L I} \sim\left\langle H_{i}^{\mathrm{int}} \frac{P^{2}}{2 m_{i}^{2} c^{2}}\right\rangle \sim \frac{\hbar \omega_{0}(2 n+1)}{m_{i} c^{2}} a_{i}^{\prime},
$$

where the perturbation calculation gives the first order modification of the energy level by $\left\langle n\left|P^{2}\right| n\right\rangle=\frac{m_{i} \hbar \omega_{0}}{2}(2 n+1), n$ is an integer and represents the energy level of the atom, and $\omega_{0}$ is the equivalent frequency of the oscillator corresponding to the energy level used experimentally.

Then, we will investigate whether the LV model in the second and third section can be put into the frame of the recent quantum formulation of EEP based on the concrete form (15) constructed by the previous suggestion [21]. While considering the LV effect, the crucial part of the Hamiltonian is expressed as [16],

$$
H_{L V}=\frac{P^{2}}{2 m_{i}}+\mu B J_{z}+\kappa J_{z}^{2}=\frac{P^{2}}{2 m_{i}}+\mu B\left(\begin{array}{cc}
\frac{1}{2} & 0 \\
0 & -\frac{1}{2}
\end{array}\right)+\kappa\left(\begin{array}{cc}
\frac{1}{4} & 0 \\
0 & \frac{1}{4}
\end{array}\right) \text {, }
$$

where the representation of spin- $\frac{1}{2}$ is taken to compare with the Hamiltonian (15). As discussed in the last section, the LV effect was designed to be measured using atomic Ramsey interferometry and the dynamical decoupling method. It is easy to see from Eqs. (15) and (17) that the above LV effect only causes the shift of energy levels but do not cause transitions to different energy levels due to the absence of off-diagonal terms. Hence, it only constitutes a classical violation of EEP.

Consider the example discussed before for $\mathrm{Yb}$ atom, and one can estimate the parameter $a_{i}^{\prime}$. As tabulated in Table I of the Ref. [16], the shift of energy level caused by LV effect is

$$
\Delta E_{i}^{L V} \sim h C_{0}^{(2)} \times 3.9 \times 10^{16} \mathrm{~J} \sim 10^{-40} \mathrm{~J}
$$

where $h$ is the Planck's constant and $C_{0}^{(2)} \sim 10^{-23}$ according to Ref. [14]. By identifing 
$\Delta E_{i}^{L V}$ with $\Delta E_{i}^{L L I}$ in Eq. (16), we obtain

$$
a_{i}^{\prime} \sim 10^{-19},
$$

where the same parameters as in Ref. [21] are adopted for the Eq. (16), i.e. $n=2$, $\mu=0.429 \mu_{N}, B=1 T, \omega_{0}=10^{4} \mathrm{~Hz}$. This result shows that the present experimental observation for the shift of energy level is less sensitive than that expected in Ref. [21] where $a_{i} \sim 1$ (the violation parameter was defined as $\left(a_{i}+\frac{1}{2}\right) \mu B$ in Ref. [21]) or $a_{i}^{\prime} \sim 10^{-24}$ (according to the definition here for the violation parameters in Eq. (15)) is taken by hand. A subtle point has to be clarified. For the definition of the previous violation parameters, i.e. $\left(a_{i}+\frac{1}{2}\right) \mu B$, it seems that the value of $a_{i}$ should be at the level of 1 which is just the value taken in Ref. [21] to estimate the transition probability. In fact, $\left(a_{i}+\frac{1}{2}\right)$ can take any value since the violation is added into the Hamitonian by hand. So it is better to introduce a new parameter $a_{i}^{\prime}$ to replace the previously whole expression $\left(a_{i}+\frac{1}{2}\right) \mu B$ without influencing any discussion about the violation of LLI in the quantum formulation of EEP. In this way, it is seen that the violation parameter $a_{i}$ that is assumed to be 1 in Ref. [21] is equivalent to $a_{i}^{\prime} \sim 10^{-24}$. Although it is smaller than that given by Eq. (19), it cannot be a constraint since the estimated transition probability is too small to be observed at the present experimental presicion to the best of our knowledge. Therefore, we can declare that the Eq. (19) represents a constraint for the violation parameter from the present experiments [14, 16], unless a better constraint is found from any other experiments.

It is noted that when we constrain the violation parameter $a_{i}^{\prime}$ with the atomic experiments related to the LV test (it is easy to repeat the above related discussion to give the constraint for the violation parameter $c_{i}^{\prime} \sim 10^{-19}$ ), we have assumed that the discussion about the $\mathrm{LV}$ is based on one specific model for LLI violation in the quantum formulation of EEP, in which

the specific model is given by the atom of spin- $\frac{1}{2}$ state placing in an external magnetic field. Whether the phenomenological description for the quantum formulation of EEP is consistent with SME theory in essence required a further investigation.

\section{CONCLUSION}

In this paper, we revisit the LV effect and its influence on the Ramsey interferometry. We provide a clear expression for the atomic Ramsey signal in the absence or presence of the 
LV effect. It is emphasized that this type of the LV effect discussed presents itself only as a local influence which limits its detectability using the experiments of quantum entanglement beyond the standard quantum limit unless a new understanding is found or suggested. The LV effect can be regarded as equivalent to a Hamiltonian proportional to the square of the angular momentum component along the z-axis, which can be compared with the recently suggested quantum formulation of the EEP. We compare the LV effect and the description for the LLI in the quantum formulation of EEP, and find that the LV effect only amounts to a kind of classical effect which cannot generate the non-local influence unitarily to change

entanglement among atoms. Based on the spin- $\frac{1}{2}$ system, we build the relation to constrain the classical violation within the new EEP test framework by the precision experiments performed to test the LV. Although the built relation requires the further confirmation, in particular in the presence of gravity, our work still provides an interesting and novel exploration for the connection between LLI violation in the quantum formulation of EEP and LV in SME.

\section{ACKNOWLEDGE}

This work is supported by National Natural Science Foundation of China (NSFC) with No. 91636213 and No. 11654001. We also want to thank Li You and Lingna Wu for their suggestions and discussions.

[1] D. Mattingly, Living Rev. Relativity 8, 5 (2005).

[2] S. Liberati, Class. Quantum Grav. 30, 133001 (2013).

[3] J. D. Tasson, Rep. Prog. Phys. 77, 062901 (2014).

[4] D. Colladay and P. McDonald, Phys. Rev. D 55, 6760 (1997).

[5] D. Colladay and P. McDonald, Phys. Rev. D 58, 116002 (1998).

[6] V. A. Kostelecký and N. Russell, Rev. Mod. Phys. 83, 11 (2011).

[7] V. A. Kostelecký and C. D. Lane, Phys. Rev. D 60, 116010 (1999).

[8] R. Bluhm, V. A. Kostelecký, N. Russell, Phys. Rev. Lett. 82, 2254 (1999).

[9] H. Müller, S. Herrmann, A. Saenz, A. Peters, and C. Lämmerzahl, Phys. Rev. D 70, 076004 
(2004).

[10] S. Herrmann, A. Senger, K. Möhle, M. Nagel, E. V. Kovalchuk, and A. Peters, Phys. Rev. D 80, 105011 (2009).

[11] M. A. Hohensee, N. Leefer, D. Budker, C. Harabati, V. A. Dzuba, and V. V. Flambaum, Phys. Rev. Lett. 111, 050401 (2013).

[12] V. A. Kostelecký, A. J. Vargas, Phys. Rev. D 92, 056002 (2015).

[13] T. Pruttivarasin, M. Ramm, S. G. Porsev, et al., Nature 517, 592 (2015).

[14] V. A. Dzuba, V. V. Flambaum, M. S. Safronova, S. G. Porsev, T. Pruttivarasin, M. A. Hohensee, and H. Häffner, Nat. Phys. 12, 465 (2016).

[15] V. V. Flambaum, Phys. Rev. Lett. 117, 072501 (2016).

[16] R. Shaniv, R. Ozeri, M. S. Safronova, S. G. Porsev, V. A. Dzuba, V. V. Flambaum, and H. Häffner, Phys. Rev. Lett. 120, 103202 (2018).

[17] C. W. Misner, K. S. Thorne, and J. A. Wheeler, Gravitation (Freeman, San Francisco, 1970).

[18] T. Damour, Class. Quantum Grav. 29, 184001 (2012).

[19] E. D. Casola, S. Liberati, and S. Sonego, Am. J. Phys. 83, 39 (2015).

[20] M. Zych and Č. Brukner, arXiv: 1502.00971 [gr-qc] (published online in Nature Physics).

[21] P. J. Orlando, R. B. Mann, K. Modi, and F. A. Pollock, Class. Quantum Grav. 33, 19LT01 (2016).

[22] V. A. Kostelecký, Phys. Rev. D 69, 105009 (2004).

[23] Michael A. Hohensee, Steven Chu, Achim Peters, and Holger Müller, Phys. Rev. Lett. 106, 151102 (2011).

[24] V. A. Kostelecký and C. D. Lane, J. Math. Phys. (N.Y.) 40, 6245 (1999).

[25] V. A. Kostelecký and J. D. Tasson, Phys. Rev. D 83, 016013 (2011).

[26] R. G. Unanyan and M. Fleischhauer, Phys. Rev. Lett. 90, 133601 (2003).

[27] S. Choi and N. P. Bigelow, Phys. Rev. A 72, 033612 (2005).

[28] S. Choi and B. Sundaram, Phys. Rev. A 77, 053613 (2008).

[29] M. A. Nielsen and I. L. Chuang, Quantum Computation and Quantum Information, (Cambridge University Press, Combridge, UK, 2000).

[30] M. Kitagawa and M. Ueda, Phys. Rev. A 47, 5138 (1993).

[31] D. J. Wineland, J. J. Bollinger, W. M. Itano, et al, Phys. Rev. A 50, 67 (1994).

[32] M. J. Halland and K. Burnett, Phys. Rev. Lett. 71, 1355 (1993). 
[33] C. K. Law, H. Pu, and N. P. Bigelow, Rev. Lett. 81, 5257 (1998).

[34] B. Lücke, M. Scherer, J. Kruse, et al., Science 334, 773 (2011).

[35] X. -Y. Luo, Y. -Q Zou, L. -N Wu, et al., Science 355, 620 (2017).

[36] Y. -Q Zou, L. -N Wu, Q. Liu, et al., PNAS 201715105 (2018).

[37] T.-L. Ho and S. K. Yip, Phys. Rev. Lett. 84, 4031 (2000).

[38] L.-N. Wu and L. You, Phys. Rev. A 93, 033608 (2016).

[39] G. Rosi, G. D’Amico, L. Cacciapuoti, F. Sorrentino, M. Prevedelli, M. Zych, C. Brukner, and G. M. Tino, Nat. Commun. 8, 15529 (2016). 